\title{
LA SCRITTURA UDIBILE - ALCUNE OSSERVAZIONI SUL ROMANZO VOCI DI DACIA MARAINI
}

\section{Su Voci}

Voci è un romanzo sulle violenze contro le donne, ma non sembra questo l'unico tema di questo pezzo di narrativa di Dacia Maraini. Innanzitutto, è un libro in cui ascoltiamo e sentiamo tutta la ricchezza dei suoi suoni, dei rumori e delle voci attraverso il muto segno della scrittura. Il perché di tale effetto sonoro e come è stato raggiunto costituiranno una parte delle riflessioni che seguono. Inoltre, quello della Maraini è un libro sul senso dell'udito, sull'importanza delle percezioni uditive nel processo del conoscere la realtà circostante e sulle possibilità di descrivere il mondo partendo in gran parte da quello che riceve l'orecchio. Infine, è anche un libro sui sensi, intesi come significati che possono essere nascosti sotto i suoni, sotto la voce ovvero nell'assenza di essa. Sono questi i temi, una volta tolto il velo della trama, che traspaiono o meglio che risuonano dalle righe di Voci.

Il libro del 1994 racconta di un'estate di Michela Canova, giornalista radiofonica, che prepara un programma sui delitti contro le donne. Si appassiona particolarmente ad un caso, quello che le è più vicino $\mathrm{e}$, in un certo senso, più familiare, il caso di Angela Bari, la sua dirimpettaia, uccisa da ignoti con venti coltellate. Michela diventa detective, deve far 'parlare' a tutti i costi la vicina di casa, prima che la sua morte diventi per sempre un numero muto fra tanti in un freddo elenco di decessi. E le voci sono quelle che la protagonista, Michela Canova, va raccogliendo con il suo registratore : le ascolta e riascolta, per scoprire l'incrinatura che, forse, nasconde una verità o una menzogna, l'accento, la titubanza, il lieve stridio, l'intonazione rivelatrice.

La protagonista costituisce l'asse intorno al quale si muove la trama e intorno al quale ruotano gli altri personaggi. Tale concetto è stato anche ribadito dalla stessa Maraini, che in una delle numerose interviste afferma : « Io parto molto dai personaggi [...]. Quando io devo scrivere un libro, di solito è un progetto attorno a un personaggio [...]. Quindi è il personaggio di solito che mi invoglia, che mi trascina, che mi spinge a scrivere un libro »(MARAINI, 1998). Ciò che rende speciale il rapporto di Michela Canova con il mondo è il senso dell'udito, il cui funzionamento le assicura un contatto davvero unico con la realtà. Per motivi legati alla sua professione o semplicemente perché è dotata di tale personalità, Michela è particolarmente sensibile ai suoni, alle voci che la raggiungono da tutte le parti e non sono solamente voci umane. Michela, si può dire, ascolta il mondo, l'udito è il suo senso più sviluppato, più acuto, ed è attraverso l'udito che esamina la realtà, giunge agli strati più profondi e più veri delle persone. A volte piccole sfumature, piccole incrinature delle voci altrui le bastano per capire il complicato groviglio di relazioni e dipendenze tra le persone. È l'udito a guidarla nella ricerca della verità e a portarla a scoprire le dinamiche delle cose. E non si tratta qui ovviamente dell'orecchio assoluto o dell'udito ipersensibile, ma abbiamo a che fare con una speciale attenzione, una attitudine al confronto del suono, delle voci e del senso che si può ricavare a forza di ascoltare. 


\section{La Maraini e i sensi}

Sicuramente il senso dell'udito, il suono, la voce interessano la scrittrice e costituiscono un argomento o forse anche un esercizio di scrittura. Sembra tanto più legittimo sostenerlo quanto si ha in vista la concezione della scrittura della Maraini. In un'intervista dichiara esplicitamente : "Per me la scrittura passa attraverso i sensi » (MARAINI, 1997). Infatti la sua prosa è stata sempre molto realistica, la sua scrittura molto sensuale. Nell'introduzione al suo libro intitolato Amata scrittura, Viviana Rosi annota : "Credo che il segreto della abilità comunicativa di Dacia Maraini, come persona e come scrittrice, stia in buona parte nella sua capacità di accogliere il mondo, di starlo a sentire, senza mai permettere al pregiudizio di sviare l'attenzione, di cancellare il rispetto dovuto agli altri, a chiunque altro » (ROSI, 2005 : 9). Forse le sue origini siciliano-cileno-inglesi, i suoi viaggi remoti (perché Dacia viaggia sempre molto) o quelli di suo padre che quando tornava dai suoi viaggi si portava dietro gli odori di : « vecchie mele [...], di biancheria usata, di capelli scaldati dal sole, di libri scartabellati, di pane secco, di scarpe vecchie, di fiori macerati, di tabacco di pipa, di balsamo della tigre contro i reumatismi » (MARAINI, $2004: 42$ ) l'hanno portata a elaborare uno stile particolarmente attento ai sensi, a quella « scrittura dei sensi che sarà - come molti hanno notato - una delle più evocative e magiche peculiarità della prosa di Dacia Maraini. Odori di mare, di grasso di maiale, di cipolla, di basilico, di erba medica, di margherite bruciate dal gelo » (DI PAOLO, 2003).

Così, non si può separare la scrittura di Dacia Maraini dai sensi. Quello che ora ci interessa in particolar modo è l'udito, uno dei due sensi più importanti se non il più importante vista l'etimologia della parola "assurdo". Essa deriva dall'accezione che il latino medievale surdus ha nell'espressione radix surda, utilizzata per tradurre l'arabo jadr asamm, che in matematica indica una radice irrazionale (ACKERMAN, 1994 : 183). L'espressione araba è a sua volta una traduzione dal greco della parola alogos che significa 'muto' oppure qualcosa di irrazionale. Da questo groviglio di significati risulta che il senso dell'udito, più che la vista, ci procura il legame razionale e logico con il mondo, e che, se la cecità permette ancora di capire le dinamiche della realtà, la sordità rompe completamente questo legame lanciandoci nell'assurdità (ACKERMAN, 1994 : 183). Basti solo pensare alle difficoltà che devono affrontare le persone sorde dalla nascita per poter entrare nell'universo delle parole e quindi poter chiamare le cose, nominare le idee, comunicare con gli altri, esprimersi. L'udito è accompagnato dalla voce, praticamente istituisce la voce, permettendo di ricevere i suoni prodotti anche da noi stessi. Sentire la propria voce significa prendere coscienza di se stessi, rendere il corpo presente a se stesso. ${ }^{103}$ «La voce è coscienza » dice Husserl (DERRIDA, 1997 : 134).

Quanto sia importante il legame tra l'udito e il nostro modo di essere al mondo lo dimostra Michela Canova, la protagonista di Voci. Michela sente e ascolta.

\section{Ascoltare Voci}

La scrittura è muta, le parole scritte, se non lette, non producono da sé nessun suono, con la voce hanno in comune soltanto il carattere temporale, se concepite

\footnotetext{
${ }^{103}$ A questo proposito si sono espressi in molti. Cfr. FLIEGER (1994 : 77-78) ; SERRES (1985 : 117) ; DUFRENNE (1987 : 63) ; DERRIDA (1997 : 131-134).
} 
nel processo dello scrivere. Ebbene, il libro Voci è un libro da ascoltare, è un libro molto rumoroso, un libro i cui suoni, rumori, voci sentiamo e ascoltiamo, percorrendo con lo sguardo i segni scritti, muti. In tal senso siamo noi, lettori ad ascoltare Voci, ma chi ascolta è anche la protagonista che sente il mondo, ascolta voci altrui, rumori dalla strada, suoni degli oggetti, cercando di dare senso alle percezioni dell'udito. Infatti ascoltare secondo la spiegazione enciclopedica significa : «mettersi in condizione di decodificare ciò che è oscuro, confuso o muto, per far apparire alla coscienza il 'di sotto' del senso » (BARTHES, HAVAS, 1977 : 984). Michela per la sua professione di giornalista radiofonica è abituata ad ascoltare e ripensare le voci. Dice :

«La mia vita oramai sembra fatta solo di voci estranee che cerco di decifrare, analizzare » (104).

Tuttavia l'acutezza di questo senso è anche radicata nella personalità, nel suo rapporto con la realtà, nell'atteggiamento del suo corpo pronto a ricevere stimoli uditivi, perché come un giorno dice :

« Mi accorgo che giro intorno al telefono cercando un pretesto per chiamare qualcuno e ascoltare una voce. Sono avida di voci, che siano leggere o pesanti, scure o chiare, le amo per la loro straordinaria capacità di farsi corpo. Mi innamoro di una voce, io prima che di una persona ; forse per questo lavoro alla radio ; o è il mio lavoro alla radio che mi porta a dare corpo alle voci, ascoltandole con carnale attenzione ? » (69).

La sua è dunque un'attitudine particolare all'ascolto. Michela ama le voci, ama ascoltarle, ne sente un bisogno si direbbe carnale, quasi « narcotico », girando intorno al telefono, cercando pretesti, comportandosi come chi è dipendente da una sostanza stupefacente, una droga che procura un momento di effimero piacere. Per Michela, "che siano leggere o pesanti », le voci sono droghe di cui ha bisogno. Per lei, le voci perdono la loro fenomenalità, la loro appariscenza temporanea, precaria. Le voci si concretizzano, si solidificano, diventano corpi, « si fanno corpi » di per sé, ma anche Michela « dà corpo » alle voci. Michela percepisce quindi le voci come sostanze, come qualcosa di duraturo e tangibile. Le voci « che si fanno corpo » diventano delle unità a sé stanti, oggetti percepibili con la vista, il tatto, l'olfatto, il gusto, ne testimoniano gli aggettivi che descrivono le voci sia in questo breve frammento sia in molti altri passi. Le voci si materializzano. Qui ci avviciniamo alla descrizione enciclopedica data alla voce da $R$. Barthes e R. Havas : «...la voce non è un soffio, bensì la materialità del corpo che sgorga dalla gola, là dove si forgia il metallo fonico » (BARTHES, HAVAS, 1977 : 988). Però per Michela le voci sembrano avere un'autonomia ancora più grande perché :

« le voci sono corpi in moto e hanno ciascuna l'ambiguità e la complessità degli organismi viventi ; belli o brutti, deboli o forti che siano, sono percorse da vene lunghissime di un azzurro che mette tenerezza, seminate di costellazioni di nei come un cielo notturno ed è difficile metterle a tacere come si fa con le parole cartacee in un libro $\gg(300)$.

Le voci possiedono un « corpo » diverso da quello che le produce. L'idea della separazione della voce dal corpo si fa netta frequentemente tra le righe del romanzo, ad esempio nel semplice commento della protagonista di un qualsiasi avvenimento quotidiano : 
« Nel silenzio della notte sento lo squillo del campanello. Mi alzo e, soprappensiero, vado al telefono, ma non si tratta di una voce che chiama bensì di un corpo che si presenta alla porta » (137)

Ne consegue una semplice regola metaforica valida per tutto il libro : la voce non necessita di un corpo e il corpo non sempre ha una voce. Infatti, è proprio il rapporto tra voce e corpo che pare intrinseco per questo testo, come lo si vedrà in seguito. La voce e il corpo saranno spesso posti in termini di contrasto e attraversando le sfumature di esso, dalla simbiosi alla disgiunzione con vari gradi mediani, si studierà la posta in gioco : la vita o la morte.

Michela, a sua volta, ascolta queste voci-corpi con « carnale attenzione ». L'attenzione è un atteggiamento della mente, un atteggiamento di concentrazione e di vigilanza della mente, « un nuovo modo di essere della coscienza di fronte ad altri oggetti »(MERLEAU-PONTY, 2003 : 14-15), ma Michela ascolta le voci con tutto il corpo, con la carne, con tutti i sensi all'erta, con tutta se stessa. Di fronte alle voci-corpi, lei stessa è un corpo. Infatti le voci, sensazioni destinate all'udito, si dirigono verso altri sensi : verso il tatto perché sono « leggere o pesanti », verso la vista perché sono « chiare o scure ». È un atteggiamento molto sensuale, carnale, della protagonista nei confronti delle voci. Michela ascolta le voci-corpi impiegando tutti i sensi, le ascolta con « carnale attenzione », e si innamora di esse. Dice : «Mi innamoro di una voce, io prima che di una persona ». Non dice che la voce è la prima cosa di cui si innamora in una persona, cosa normale, ma che si innamora di una voce più velocemente, prima ancora che riesca a innamorarsi di una persona. Sono le voci, per lei, a essere affascinanti, sensuali, ad avere una personalità di cui innamorarsi. Il suo modo di ascoltare è quindi anche molto emotivo. Le voci agiscono non solo sul suo orecchio, molto di più, agiscono sugli altri sensi, su tutto il suo corpo che ascolta in attesa di tali sensazioni. C'è un'altra parola che, nel brano riportato, rimanda alla carnalità. Michela dicendo di se stessa : «Sono avida di voci », così come si è spesso avidi di cibo o di bevande, sposta il suo interesse per le voci verso il piano corporale, verso quell'abisso del corpo dove finisce tutto ciò che viene ingoiato. Lo stesso atteggiamento carnale, anzi consumistico, della protagonista nel confronto delle voci viene ribadito con parole più suggestive ancora in un altro posto :

« Mi piacerebbe dimenticarle, ma il mio orecchio ha una voracità animalesca, e come un maiale, grufola cacciando il naso fra i rifiuti sonori, mandando giù con disinvoltura frasi fatte, luoghi comuni, giudizi preziosi e citazioni dotte così come mi arrivano dai microfoni, aspettando poi che lo stomaco faccia le sue drastiche selezioni » (13).

Gli orecchi di Michela non si saziano mai di voci al punto tale che talvolta in esse, perfino in quelle immaginate, risuona il peso degli avvenimenti drammatici realmente accaduti :

«Le voci in questi giorni si sono moltiplicate pericolosamente, hanno toni minacciosi come se una eco dell'assassinio di Angela Bari riverberasse su tutto ciò che avvicino » (21).

Infatti, tra le voci divorate avidamente da Michela ovvero tra quelle ascoltate e considerate da lei si possono individuare, grosso modo, due gruppi.

Il primo è molto individuale. Si tratta di voci irreali, forse frutto della sua immaginazione che le fa sentire voci umanizzate, personalizzate, laddove ci sono solo rumori indecifrabili o niente. Sono voci che parlano in lei. Qui con Antonio 
Tabucchi possiamo constatare che «siamo nel regno degli acúsmata: essere in stato acusmatico, o d'incantamento, è percepire qualcosa che dipende dall'allucinazione sonora. [...] Il regno dell'acúsmaton è vietato all'estraneo. Appartiene soltanto a chi può ascoltare le sue voci mentali ${ }^{104}$. Infatti, sin dalle prime pagine Michela ci trascina nel suo mondo interno popolato da voci svariate degli oggetti :

«Gli oggetti stamattina parlano, parlano e sembrano tutti spinti da una urgenza gregaria.

Anche il sapone sembra avere una voce, rauca e soffiata, come di uno che è stato operato alla gola. Quanto chiacchierano gli oggetti !» (12) ; o quelle delle piante :

« Non amano stare sole, le mie piante e me lo dicono con voci chiocce in un sussurro dietro le spalle » (9);

o le voci delle scarpe da tennis della ragazza uccisa :

« Scarpe leggere da tennis... anche loro dicono qualcosa, ma cosa ?» (12);

o le voci degli animali abbattuti appesi dal macellaio :

« mi sembra di sentire un mormorio alle mie spalle. Le testine appese sono scosse da risatine sinistre, singulti e squittii » (20).

Michela ascolta la voce del letto :

« Il letto intanto mi sta dicendo qualcosa, ha una voce bassa e soffocata, mi pare che dica 'dammi le tue ossa', ma non so » (69);

e di altri oggetti di casa :

«Anche il pentolino sul fuoco si è messo a parlare, anzi a canticchiare, proprio come Stefana dice che faceva Angela Bari a casa sua. Alle due voci si aggiunge quella della trottola che faccio girare sul tavolo davanti a me : uno zufolio ritmato che sembra volerti ipnotizzare.

A quest'ora della notte gli oggetti diventano impudenti : chiacchierano, cantano, vociferano » $(70)$;

oppure :

« Il lavello ha una voce cristallina, petulante : quando apro il rubinetto dopo un giorno di assenza emette un leggero singulto festoso ; soffre di "secchezza delle fauci": [...]»;

o ancora :

« I giornali alle mie spalle stanno facendo un gran chiasso » (127).

Michela nota anche degli insoliti silenzi, la mancanza delle voci :

«Gli oggetti sono stranamente silenziosi oggi a casa mia, che stia cambiando il tempo ? Che sia questa mancanza assoluta di vento, questa quiete afosa e tetra ?» (237).

Queste voci liberano dalla prigione del silenzio il mondo delle cose con cui la protagonista si unisce attraverso la sua sensibilità uditiva. Infatti i brani riportati non sono interessanti solo come testimonianza della percezione uditiva anormale della protagonista, della sua voracità nei confronti dell'udibile ma vale anche la

\footnotetext{
104 TABUCCHI (2003 : 35-36). L'autore aggiunge : « Ma quel termine che designa le voci che noi crediamo di sentire ha origini molto antiche : per i Padri della Chiesa erano le voci degli angeli, quando si fanno sentire dentro di noi. Si dice ad esempio che santa Cecilia, durante il martirio, sentisse il canto degli angeli dentro di lei ; ed è per questo che è stata eletta a santa protettrice della musica ».
} 
pena considerare gli oggetti che emettono la voce, nonché riflettere sulla loro posizione in questo testo. Sembra applicabile la tesi di Przemysław Czapliński, il quale sostiene che le cose nella narrativa degli anni '90 parlano, hanno qualcosa da dire, comunicano qualcosa e quindi significano, diventano semiotiche. Le cose prendono la parola soprattutto quando le riportiamo alle persone e quando le trattiamo come un'espressione della vita, che sia pubblica o privata, esteriore o interiore. Le cose dunque non si riducono a un problema mimetico nella letteratura ma vengono lette come un problema semiotico. (CZAPLIŃSKI, 1999 : 209-210). Le cose che parlano a Michela pare che abbiano quindi una risonanza : hanno la voce e parlano letteralmente, sentite da Michela, e poi parlano nel senso usato da Czapliński, comunicano e significano, come lo fanno d'altronde anche le cose prive di voce : ad es. il suo registratore, la sua macchina color ciliegia che « sembra offesa e muta ma poi con un colpo di tosse e molti brontolii, si decide a partire » (p. 15), e così le cose parlano di Michela. Parlano della sua sensibilità, della sua solitudine, della sua paura e della sua lotta contro la realtà. Tra le cose a cui presta l'orecchio Michela ce n'è una che parla della ragazza uccisa, sono le sue scarpe da tennis azzurre le quali apparendo più volte nel romanzo acquistano un valore simbolico. Diventano simbolo della leggerezza del suo essere, ma anche della sua morte inutile e brutale.

Michela ascolta le voci delle cose facendole vivere e significare nel tessuto del romanzo, rendendole autonome unità narrative. La sua è la relazione di empatia verso le cose ovvero un'attitudine segnata dalla volontà di provare quello che prova ciò che è privo di linguaggi umani destinati a esprimere (CZAPLIŃSKI, 1999 : 234).

Michela ascolta anche le voci dei morti, soprattutto quella del padre con cui riesce a instaurare un rapporto tenero e premuroso.

«Spengo la luce, mi giro su un fianco, ma il sonno fatica a chiudermi gli occhi. Sento la voce di mio padre che, tornando la notte, si chinava sul mio letto dicendo : "Ancora gli occhi spalancati, Michela ?" [...] "Non ho più quindici anni, papà" "Ma se sei una ragazzina ! Vuoi che ti dia il bacio della buonanotte ?" "No papà, ricordati che sei morto e le tue mani non possono toccarmi" » (29-30).

Invece la voce della madre, evocata dall'al di là ${ }^{105}$, tradisce un rapporto difficoltoso tra madre e figlia, un rapporto pieno di incomprensioni e divergenze.

" "I ragni, bisogna volergli bene", è la voce di mia madre che mi parla all'orecchio ; chissà perché l'ho tanto odiata quella voce, al punto di modificare la mia e renderla irriconoscibile, lontana da ogni area familiare. Era la voce del buon senso quotidiano, abitata da oscuri timori che io rifiutavo anche solo di indovinare, una voce non solo educata ma domata. $\mathrm{Ci}$ sono voluti anni di radio per imparare a rendere naturale l'estremo artificio linguistico del parlato giornaliero » (166).

Le presenza dei genitori morti è possibile solo attraverso la voce. Essa diventa allora un medium quasi spiritistico.

Poi si fa ascoltare la voce ammonitrice di suor Esterina :

\footnotetext{
${ }^{105}$ A questo proposito TABUCCHI (2003: 23) ricorda : « evocare significa anche richiamare qualcuno dal regno dei trapassati per mezzo delle facoltà medianiche : il che riporta all'esoterismo contenuto nella misteriosa forza della voce di Orfeo ».
} 
« Nel dormiveglia sento la voce di suor Esterina che dice : “È l'anima di Angela Bari che non ha pace, povera colombella chissà quanto patisce non trovando una finestra aperta da cui volarsene dove Cristo l'aspetta.... Vai a liberare quella povera colombella, se no sarai tu la colpevole verso Dio, vai, corri !"’ $\gg(71)$.

Michela sente perfino le voci meno esplicite delle molte ragazze uccise che conosce solamente attraverso i fascicoli della polizia e sulle quali deve preparare un programma :

«Sono sola col mio registratore e tante voci che si incalzano, premono. Fanno un gran chiasso queste morte ammazzate e non so da chi cominciare » $(85)$.

Sono voci mentali, appartenute alla sensibile psiche e all'orecchio acuto di Michela.

Al secondo gruppo invece appartengono le voci che costituiscono un paesaggio acustico comune, sentito da tutti quelli, il cui udito funziona normalmente permettendo loro di sentire suoni della natura e rumori quotidiani di diverso genere : i rumori prodotti dai raccoglitori dell'immondizia, dalle scarpe, dalle porte che si aprono e si chiudono, dall'ascensore, dalle serrature, dai campanelli. Comunque le voci più risonanti di questo gruppo e quelle che costituiscono il tessuto più fitto del libro sono le voci delle persone che Michela conosce $o$, più spesso, di quelle che incontra nel corso delle sue indagini. Nel secondo caso la voce, la sua tonalità, timbro, intonazione, espressione diventano informazioni precise, cosa su cui rifletteremo in seguito. Le voci in gran parte risuonano nei dialoghi che si svolgono senza nessun referente, nel presente, in diretta, proprio nel momento in cui li leggiamo. Il momento in cui vengono pronunciate le parole, in cui «viene emessa la voce » coincide con il momento della lettura, coincide con il presente. Tuttavia, in molti casi, le voci umane ci giungono da fonti diverse da quelle naturali, ci giungono mediante un apparecchio artificiale che le manipola, trasforma, immobilizza e le dota di un supporto materiale. I dialoghi hanno molto spesso la forma di comunicazioni telefoniche e allora la persona che produce la voce è soltanto udibile e non visibile. Cosa interessante è che, tra le undici persone che la protagonista incontra facendo la sua indagine, il primo contatto con cinque di loro avviene attraverso il filo telefonico e quindi unicamente con la voce e le facoltà della percezione uditiva. Il momento del primo incontro con una di esse ha luogo tramite il citofono e quindi di nuovo un apparecchio che agevola lo scambio di voci ma impedisce il conoscersi attraverso gli altri sensi. Mentre il rapporto con il fidanzato, la persona più cara e amata, nell'arco di due mesi, tempo in cui si svolge la trama del romanzo, si realizza unicamente a voce : parlando al telefono, lasciando messaggi sulla segreteria telefonica oppure come mandando comunicazioni registrate su un nastro. La voce del professor Baldi, psicologo esperto della radio in cui lavora la protagonista, viene spesso modificata con mezzi tecnici : « Tolgo del tutto la musica, schiarisco per quanto possibile la voce del professore col rischio di deformare quella dell' ascoltatore, tengo due dita sul miscelatore cercando di non scompensare troppo il livello dei suoni » (55). La segreteria telefonica e il nastro sono anche veicoli postumi della voce di Angela, la ragazza uccisa. Si può supporre così che la voce umana non sarebbe più « ideale », nel senso fenomenologico poiché non sempre si riduce alla pura trasparenza. Il parlare 
umano non può essere sempre considerato come atto vitale, atto della presenza della vita (DERRIDA, 1997 : 117-147).

Tale struttura vocale del libro comporta due ulteriori riflessioni : sul rapporto voce-presenza-assenza, e sulle possibilità dell'udito nel conoscere l'altro e quindi sul valore epistemologico dei sensi.

\section{La voce e il corpo}

Nel romanzo Voci si delinea una struttura graduale del rapporto voce-corpo che si estende tra due poli : presenza e assenza. Si tratta da una parte della vitale presenza della voce ${ }^{106}$ associata alla presenza della persona, del suo corpo che produce la voce, e dall'altra, della situazione in cui la voce è priva di corpo, oppure non ci sono né corpo né voce ma rimangono le rappresentazioni. Mentre il primo polo indica la vita, il secondo designa la morte. Questa struttura metaforica viene espressa attraverso le relazioni che la protagonista instaura con altri protagonisti del romanzo. Il primo grado di questa scala si realizza nei rapporti con le persone che, si potrebbe dire, sono dalla stessa parte della vita, con le persone con cui Michela mantiene relazioni sane, buone e creative, non di rado amichevoli, come con l'avvocato Merli, con la commissaria Adele Sòfia, o come con Titinnanzi, un collega della radio, oppure le relazioni di convivenza condominiale con la portiera Stefana Mario e suo marito. I rapporti di Michela con queste persone si svolgono quasi sempre attraverso dialoghi diretti, senza nessun intermediario artificiale. Sono rapporti caratterizzati dalla positività vitale in cui la presenza della voce si abbina alla presenza, nel tempo e nello spazio, di tutta la persona, del corpo che produce la voce. I rapporti si basano sulla sincerità e sulla verità. A un grado superiore su questa scala si situano le relazioni in cui la presenza della voce, pronunciata nel tempo reale del contatto non si riscontra con la presenza, nello spazio, della persona stessa, del corpo, nascosto da un apparecchio da cui passa la voce : telefono, citofono o apparecchiature della radio. Tale rapporto, segnato dalla distanza reale della persona come pure dalla falsità provocata dalle apparecchiature che modificano il suono, avviene soprattutto con le persone con cui Michela ha relazioni difficili, ipocritiche o false come quella con il fidanzato Marco che la tradiva e quindi le mentiva ; con il professor Baldei, uno psicologo incompetente della radio ; con Sabrina, prostituta e amica della ragazza uccisa, che mentiva a Michela e la ingannava ; o con Glauco Elia, patrigno nonché assassino della ragazza uccisa. Su questi rapporti, segnati dalla distanza spaziale del corpo che produce la voce e basati sulla falsità, si stende spesso l'ombra della morte di Angela. Si tratta di personaggi che, in diverse fasi dell'indagine svolta da Michela, erano collegati con la morte della ragazza. Le voci di Sabrina e di Glauco Elia venivano anche registrate su un nastro il quale costituisce un'altra degradazione della presenza del corpo che produce la voce spostando l'accento verso l'assenza. Le registrazioni permettono di preservare la voce e renderla presente mentre il corpo è assente sia nel tempo che nello spazio. Infatti, qui cominciamo ad avvicinarci all'ambito della morte perché sia Sabrina che Glauco si suicidano, mentre Marco che registra la sua voce su un nastro e anche sulla segreteria telefonica, era sospettato dell'omicidio di Angela. Pure la

\footnotetext{
${ }^{106}$ La voce è il principio della vita, il principio della creazione. In principio erat Verbum. Cfr. TABUCCHI, $2003: 23-24$.
} 
ragazza uccisa ha registrato la propria voce su un nastro e sulla segreteria telefonica, come si è scoperto dopo la sua uccisione. Tali registrazioni della voce, a cui non corrisponde la presenza di un corpo dall'altra parte dell'apparecchiatura, segnano dunque il terreno della morte. La voce da sola non riesce a sorreggere la vita. Il grado successivo sono le voci dei morti sentite da Michela : la voce di suo padre, sua madre, di una suora, menzionate prima. Sono voci che sono presenti nell'immaginazione di Michela, ma sono senza corpi, senza presenza del corpo, sono, come dice Tabucchi voci che compensano le assenze, ma non le possono trasformare in presenze (TABUCCHI, 2003 : 20). Vi sono altre due situazioni che concludono simbolicamente questa gradazione tra la vita e la morte espresse attraverso la presenza o l'assenza della voce o del corpo. Michela, che per il suo programma conosce molti delitti contro le donne, guarda le loro foto, guarda la foto di Angela. Vi sono corpi silenziosi, ma non sono corpi veri, sono tracce delle persone, sono rappresentazioni dei corpi una volta vivi, assenze mute, che conservano in sé un elemento di vita, ricordo del passato. E infine una scultura, opera di Glauco, artista e al contempo assassino, una statua che rappresenta la sua figliastra alla quale lui stesso ha tolto la vita. Un corpo di pietra muto che vuole rappresentare una persona morta. L'assenza della voce s'abbina all'assenza del corpo. L'idea della vita vi è esclusa mentre compare quella della morte. Stranamente, è l'arte a contrastare la vita.

\section{L'udito come strumento di conoscenza : tra la verità e la menzogna}

I sensi sono canali che ci permettono il contatto con il mondo esteriore oppure, come direbbe M. Merleau-Ponty, sono luoghi in cui il nostro corpo si unisce con il mondo sensibile (MERLEAU-PONTY, 2003 : 277-322). I sensi ci assicurano diverse esperienze, fonte dei piaceri sensuali, tra cui anche quelli estetici. D'altro canto grazie ai sensi è possibile la conoscenza del mondo, è dunque innegabile il loro ruolo epistemologico (WILKOSZEWSKA, 1998 : 325). L'udito tra i sensi è quello che assicura il legame più forte e logico con la realtà (cfr. ACKERMAN, 1994 : 11). Sembra siano queste le verità che Dacia Maraini vuole far trapelare dalle pagine del romanzo Voci.

Tra i diversi stimoli sensoriali (visivi, uditivi, olfattivi ecc.) in base ai quali viene descritto il mondo rappresentato (personaggi, paesaggi, cose, con tutte le loro diversità, sfumature e somiglianze) nella narrativa di solito prevalgono quelli visivi, cosa che sembra conforme alla comune esperienza quotidiana.

La vista è un senso potente perché nutrito da una ricchezza enorme di sensazioni, trasformate in seguito in esperienza percettiva, ma la sua precedenza rispetto agli altri sensi non sta solo nell'abbondanza del campo in cui essa opera (perché anche gli altri sensi sono in continuo interagire con il sensibile) bensì nel linguaggio umano che riesce a denominare queste esperienze, rendendole comuni. È molto più facile trovare parole adeguate per descrivere l'aspetto di un albero che quelle per descrivere il suo odore. Questa è forse la ragione per cui la narrativa ha un debole per il visibile, per il quale ha sempre mostrato predilezione. A partire da Aristotele, che nella sua opera De Anima presenta in ordine di importanza conoscitiva i cinque sensi cominciando dalla vista, si stabilì la tradizione antica che dà precedenza al vedere nel processo conoscitivo. Diversamente, nella tradizione ebraica si privilegiava l'udito, essendo la Rivelazione « un evento acustico » (SCHOLEM, $1970: 11)$. Il valore conoscitivo della vista risulta dal suo 
legame con l'intelligenza, le cui tracce linguistiche sono evidenti nelle espressioni con cui descriviamo processi conoscitivi e comunicativi ricorrendo alla vista e allo sguardo, per esempio : « vederci chiaro », « illuminazione », ecc. (CACCIARI, 2002 : 5). Sembrano confermarlo Miller e Johnson-Laird che affermano che il nostro modello del mondo esterno, nella misura in cui lo riteniamo basato sulla percezione, deriva principalmente da quello che vediamo (MILLER, JOHNSONLAIRD, 1976 : 618).

Nel libro Voci Maraini sembra voler sfidare questa regola cercando di dare precedenza all'udito. Così, in proporzioni rovesciate, viene maggiormente descritto non il visibile ma l'udibile e, come se fossimo ciechi, siamo guidati, attraverso la realtà romanzesca, dai nostri orecchi. Le percezioni visive invece risultano spesso ingannevoli e non si può affidare loro la conoscenza. Ne testimoniano i confronti di esperienze visive con quelle uditive, come per esempio l'incontro con Augusta Bari Elia, la madre della ragazza uccisa che, a prima vista, appare come una donna distinta, elegante e sensuale :

«Quando alzo gli occhi la vedo : una bella donna snella ed elegante. Se ne sta in cima ad una larga scala che si apre un ventaglio [...]. La donna è interamente vestita di verde, perfino le scarpe e i guanti di un bel colore smeraldo [...]. Seguo, affascinata, le lunghe mani guantate di verde che si muovono nervose. Da vicino, la magnifica faccia appare un poco rigida e cartacea, solo le labbra sono mobili e sensuali, dipinte di un rosso carico, sangue di bue » (109-110).

Ma solamente quello che viene udito e registrato lascerà trapelare da dietro le quinte dell' apparenza una sottile luce sull'essenza della personalità di una donna determinata, sicura di sé e manierata :

«Alla moviola taglio e monto i nastri registrati, riascolto la voce di Augusta Bari Elia, le sue frasi smozzicate, gli schiocchi della lingua nel succhiare i cioccolatini. Curioso come fossi distratta dai lunghi guanti verdi e dall'abilità di quelle mani da lucertola. Riascoltata in studio la sua voce suona meno incrinata e incerta, quasi fluida nonostante le tante interruzioni, animata da una folle determinazione di fondo » (119).

Così conosciamo il mondo rappresentato attraverso la narrazione della protagonista concentrata sulle percezioni uditive. Così, la sonorità è la principale caratteristica di questo mondo. I suoni, i rumori, le voci costituiscono il materiale informativo per guidarci nella realtà rappresentata. La protagonista interpreta $\mathrm{o}$ descrive le dinamiche degli avvenimenti in base ai rumori che la raggiungono dalla strada, dal corridoio, dalla scala, descrive le voci delle persone con cui parla al telefono, cosa che, curiosamente, succede molto spesso nel romanzo Voci. Gli esempi sono numerosi. Eccone alcuni :

«Dal fondo dell'appartamento sento arrivare delle voci e, subito dopo vedo davanti la faccia di Stefania dai grandi occhi dolenti » (8) ;

«La sento scendere i gradini, rapida, con le scarpe di pezza che emettono appena un leggero tonfo smorzato » (9);

«A volte del dormiveglia sentivo la sua porta che si chiudeva con un tonfo, e la chiave che girava nella toppa con insistenza, trac, trac, trac. Anche le persiane venivano sprangate con energia, le sentivo sbatacchiare bruscamente sia di sera che di giorno » (10);

«Sentivo delle voci al di là del muro » (13); 
«Sento un parlottare fitto fitto e poi delle risatine. La voce fresca e squillante di Ludovica torna dentro la cornetta. [...] Ancora una parlottare, una ridacchiare e poi la voce gentile di Ludovica » (46);

«Vengo investita da altre voci, più vicine e vive. Fermo il registratore. Nella camera accanto stanno litigando rabbiosamente un uomo e una donna. Un uomo e una donna dalle molte abitudini in comune; lo si capisce dal tono sciatto e rancoroso delle voci : quella di lei insistente, petulante ; quella di lui sprezzante, opaca, annoiata » (104) ;

« Nel silenzio della notte sento lo squillo del campanello » (137);

« Appena chiusa la porta, sento dei passi per le scale... I passi si fanno più vicini, stanno raggiungendo l'ultimo piano... Sento i passi fermarsi davanti alla porta... un momento dopo i passi si allontanano, ma adesso la persona è di fronte alla porta di Angela Bari » (143);

« È notte. Per strada si sentono i tonfi dei raccoglitori di immondizia : un cassonetto viene sollevato, rovesciato nel camion e posato per terra da due bracci di ferro. Un cassonetto, due cassonetti, tre cassonetti, il camion si sposta lentamente verso il fondo della strada e il rumore si allontana assieme alle voci giovani dei netturbini che chiacchierano allegramente nella note silenziosa » (272);

« Nel silenzio che segue l'allontanarsi del camion comunale sento squillare il telefono $»(273)$.

Gli esempi si potrebbero ancora moltiplicare, ma quelli sopra elencati bastano per osservare che si tratta di semplici descrizioni dell'esperienza uditiva dal vivo, per lo più non modificata da nessun mezzo, che serve a Michela a decifrare e interpretare la realtà nel suo agire.

Talvolta comunque, soprattutto quando abbiamo a che fare con le voci umane, ascoltate spesso durante le conversazioni telefoniche, la protagonista non si limita ad annotarle o descriverle, ma esse le svelano gli stati d'animo, le intenzioni delle persone che parlano o perfino le loro personalità. La consapevolezza e la conoscenza dell'altro passano attraverso l'orecchio. Michela non vede persone arrabbiate, irritate, tranquille, nervose, inquiete, indecise, sospettose, insicure, abbattute, tristi, allegre, persone in diversi stati psichici, persone in diverse reazioni, con diversi atteggiamenti nei suoi confronti, anche quando esse non stanno dall'altra parte del filo, ma davanti ai suoi occhi, invece sente il loro carattere o umore :

« la voce al di là del vetro suona stizzosa, irata » (31);

«Mi risponde una voce calma, gentile» (34);

«Deve essere giovane, ha una voce aspra e vigorosa » (56);

«La sua voce, nella foga, si fa meno pigra e molle, con una punta di passione che non gli appartiene. Lo ascolto ammirata » (61) ;

«... La voce affettuosa appena incrinata dallo stupore mattutino... La voce altalenante... » (88).

«C'è una impudenza nella sua voce che mi esaspera ; una volontà di fare teatro a tutti i costi. [...] La voce suona lontana e titubante » (163);

«Una voce quasi supplice, dolcissima. Capisco : sta rovesciando le parti ...» (164);

« È Adele Sòfia che ha una voce lugubre, spenta » (176);

«Chiamo l'avvocato Merli. Mi risponde una voce flebile, sfiatata » (198); 
«La sua voce [i.e. di Lodovica] ha perso le incertezze, le lacerazioni, le cadute delle altre volte ; ora sgorga come un fiume in piena e non riuscirei anche volendo a fermarla » (268).

Ecco alcuni esempi del fatto che Michela riesce a entrare nei meandri dell' anima servendosi, come strumento di conoscenza, dei suoi orecchi che captano le minime sfumature della voce, le quali, nello stesso momento, vengono tradotte da lei in informazioni precise quali sentimenti, intenzioni, umori, stati d'animo degli altri.

L'orecchio di Michela assorbe tutto. Oltre ai momentanei stati d'animo decifra ciò che nella voce viene impresso dai tratti caratteriali individuali. Non conoscendo qualcuno né di persona, né di vista, è in grado di ricavare solo dalla voce informazioni sorprendentemente abbondanti e precise. Tale è ad esempio il caso del professor Baldi :

« Di persona non l'ho mai visto ; conosco solo la sua voce al telefono, schiacciata e nello stesso tempo amplificata dal mezzo meccanico. Chissà se è alto, basso, bruno o biondo. Non so niente di lui eppure mi sembra di conoscerlo bene perché la voce lo rivela, come fosse nudo al di là del filo : un uomo pacato, gentile, pigro, si direbbe, dall'intelligenza capziosa e lenta, una buona capacità di analisi e qualche tendenza, sempre per pigrizia, al cinismo. Ma ha un chiaro ascendente sugli ascoltatori perché lo chiamano in molti. Il suo segreto è un misto di severità provocatoria e di svagatezza materna. La cosa che più mi piace di lui è una certa risatina imprevista che ogni tanto salta fuori senza che lo voglia, come un soprassalto di bambinesca allegria. Che fa a pugni con la sua abituale voce scorrevole e savia, abituata a dare consigli, a impartire lezioni, a consegnare ricette, a fare diagnosi a distanza » (56-57);

o quello di Angela, la dirimpettaia uccisa, che Michela conosceva appena :

« La voce, quelle rare volte che l'ho sentita, mi è sembrata velata, come di chi tema di esporsi e infastidire, una voce piegata su se stessa, resa opaca dalla ritrosia, con dei guizzi inaspettati di ardimento e di allegria » (9).

Ascoltare la voce è l'unico modo per avvicinarsi al personaggio di secondo piano Marta Girardengo :

« È Marta Girardengo che parla. Noto che ha una voce di gola, molto controllata, una voce che ha cancellato i suoi echi interni, tenuta ben stretta anche quando non ce ne sarebbe bisogno. Ora ride e la sua risata, anziché sciogliere la voce, la rende più secca e ingolata come se ridere le costasse uno strappo profondo » (156-157).

Sentire e riascoltare la voce ha permesso alla protagonista di scoprire un aspetto dell'uccisore della ragazza, suo padrino, Glauco Elia :

«Ripenso alla voce che mi ha risposto al telefono : disponibile, ricercata, chiara e pulita, quasi radiofonica ; una voce di persona colta, tollerante e ironica, con qualche piccola spina di dileggio » (222);

« La sua voce ha preso un andamento musicale, me ne accorgo solo ora, come se cantasse, una melodia interna che solo i grandi seduttori sanno produrre a volontà » $(230)$;

«...la voce sensuale e corposa di Glauco Elia » (280).

Al primo ascolto, quindi, Glauco si fa conoscere come una persona gentile, colta, affidabile, forse intelligente e aperta, un uomo con un certo fascino. Non sempre comunque possiamo fidarci delle prime, immediate, consapevolezze raggiunteci 
attraverso l'orecchio come lo provano le registrazioni delle voci che Michela ascolta e riascolta, le quali, a differenza delle voci dal vivo, conservano anche ciò che sfugge mentre si è concentrati sul senso delle parole pronunciate, mentre si è depistati dalle percezioni della vista o quelle degli altri sensi. Le registrazioni hanno il potere di restituire all'orecchio le informazioni perdute, completare e arricchire la conoscenza oppure sviarla.

Le voci registrate, riascoltate e analizzate da Michela sono quelle di Angela, la ragazza uccisa ; di Carlo, suo fidanzato; di Augusta Bari Elia, sua madre; di Lodovica, sua sorella; di Glauco Elia, suo padrino; ma anche di Marco, il fidanzato di Michela e il primo sospettato. Si tratta delle voci aggrovigliate di più attorno al mistero della vita e della morte di Angela, per cui risultano più interessanti dal punto di vista della verità ricercata da Michela :

«Ogni tanto prendo il registratore e riascolto le voci che mi hanno tenuto compagnia per due mesi : quella di Angela che racconta le sue storie di re crudeli e figlie in fuga. Fuori dagli altoparlanti sofisticati della radio, suona rauca, strappata e infantile ; sembra la voce di una persona che non ama la sua voce e pensa di regalarla con cautela, quasi scusandosi del regalo nel momento che lo fa » (251);

« La voce di Giulio continua a scorrere, incantatrice. C'è tanta volontà di piacere nel suo discorso, me ne accorgo dalla lentezza con cui sciorina le frasi come fossero arazzi da mettere in vendita, mostrando la ricca consistenza del tessuto, la bellezza del disegno, lo splendore della trama. E come indugia sulle vocali finali e come soffia serpentino dentro il microfono quasi volesse trangugiarlo. [...] La squallida stanza della pensione Raffaello viene invasa dalla voce suadente, fragile e nello stesso tempo volitiva di Giulio Carlini. Mi chiedo se la seduzione di questa voce nasca dal ritmo interiore delle frasi sapientemente inanellate e distese, oppure dallo spazio intelligente che interpola tra parola e parola. [...]

Dentro la voce di Giulio Carlini si entra con piede leggero, incuriositi. Eppure c'è qualcosa che mi mette in allarme : che sia l'incrinatura appena percettibile dei toni alti che sfocia in un inaspettato stridore ? O la leggera increspatura della grana vocale che sembra nascondere una insidia, un trabocchetto ? » (104-105);

«Alla moviola taglio e monto i nastri registrati, riascolto la voce di Augusta Bari Elia, le sue frasi smozzicate, gli schiocchi della lingua nel succhiare i cioccolatini. Curioso come fossi distratta dai lunghi guanti verdi e dall'abilità di quelle mani da lucertola. Riascoltata in studio la sua voce suona meno incrinata e incerta, quasi fluida nonostante le tante interruzioni, animata da una folle determinazione di fondo.

La voce di Ludovica, ascoltata accanto a quella della madre, appare goffa e mascherata : $\mathrm{i}$ toni sono acuti e rivelano improvvisi precipizi, voragini di inquietudine $\gg(119)$.

Nelle voci registrate Michela rileva ciò che le parole non dicono e ciò che talvolta contraddicono, le menzogne :

«Anche la sua voce [i.e. di Marco], riascoltandola a mente fredda, dice molte più cose di quante dicano le sue parole. Dice di un distacco avvenuto chissà dove, un distacco che l'ha portato a formarsi un ritmo e un timbro di voce che non sono quelli che avevo conosciuti e amati, ma 
quando è avvenuto il guasto ? Una voce profondamente stanca che ha imparato a reinventarsi per sfuggire a questa stanchezza, per mascherarla. Una voce che da coetanea è diventata paterna. $\mathrm{E}$ io mi sono accorta della metamorfosi » (256);

« Apro il Nagra e ascolto le parole di Elia. A sentirla su nastro, la voce suona artefatta, come un ferro battuto e ribattuto a caldo, fino a tirarne la forma voluta. Dal vivo sembrava più arresa e sincera ; evidentemente il suo corpo mi mandava dei segnali diversi da quelli della sua voce. Lasciata sola, torna quella che avrei potuto ascoltare se avessi chiuso gli occhi : una voce circospetta e studiata, con una intenzione disperata di seduzione $\gg(235)$.

La voce da sola dice più di quanto dicano le parole e di quanto dica il corpo. Il significante ha più senso del significato, se ci riferiamo a quanto detto sul segno fonico da DERRIDA (1997 : 127-128). Nella voce si nasconde la grana della verità per chi vuole sentirla.

«Ogni voce ha il timbro della verità, che non sempre coincide con quella

logica delle cause e degli effetti cara al giudice Boni e alla commissaria

Adele Sòfia » (300).

È così forse perché la voce fa parte del corpo che è individuale, costituisce una parte integrante dell'identità personale. La voce è unica, irrepetibile, in essa si svela la nostra personalità. Ma ciò comporta d'altro canto che imitando la voce altrui possiamo prendere la sua identità. Lo illustra bene un racconto metaforico di Angela su un padre e una figlia, registrato d'altronde su una cassetta. Il padre, per costringere la figlia a restare a casa, le spezza le gambe e per controllarla le manda una civetta. La ragazza riesce a ingannare il padre insegnando alla civetta come imitare la sua voce e poi legandosi le gambe scappa, lasciando al posto suo la civetta che attraverso la porta assicura il padre della presenza della figlia (p. 188). La voce ha sostituito la persona. La civetta imitando la voce della ragazza è diventata in parte lei stessa, ha ricevuto un po' della sua identità. Per il padre che ascoltava la voce della figlia dietro la porta, la civetta è diventata sua figlia.

\section{Voci e scrittura}

Una riflessione che in questo ambito non può essere omessa e alla quale ci induce la scrittrice stessa con le due ultime frasi del libro è quella che riguarda il rapporto tra voci e scrittura. Infatti è una riflessione che viene spontanea dopo la lettura di un libro che vuole conciliare l'inconciliabile, vuole trasformare le voci che sono di natura effimera, aerea e corporea - in neri segni scritti che sono materiali, cartacei e duraturi. Il libro della Maraini vuole rinchiudere nell' anonimato muto e nel finito della scrittura il mondo dell'udibile personale e sfumato. Attraverso i segni intelligibili della scrittura vuole riprodurre percezioni sensuali dell'udito.

Su tale opposizione tra ciò che orale (e quindi udibile) e ciò che è scritto (e quindi visibile), opposizione di lunga tradizione, si pronuncia Walter J. ONG (1986) analizzando il lungo processo di trasformazione della nostra cultura, da orale a scritta, con tutte le cause e conseguenze di questo processo. Secondo Ong la creazione della scrittura fu causata dal forte desiderio di fermare il suono che si svolgeva nel tempo, di rinchiudere dunque le parole che « volavano via ». L'invenzione della scrittura riduce il suono dinamico a uno spazio tacito, aprendo 
così lunghi secoli di cultura scritta e stampata che isolava le persone. Mentre il suono dal carattere temporale penetrava l'altro, uscendo dall'interno era indirizzato verso l'interno altrui, svelava la personalità, la coscienza. Ascoltare univa, e vedere distingueva. Lo sviluppo tecnologico paradossalmente ci riporta indietro verso l'udibile e l'orale. Il telefono, la radio, la televisione, e ora anche in parte internet, ci restituiscono l'oralità, nei confronti della quale sembra che non sappiamo come comportarci : accettarla e viverla, oppure ripudiarla e voltarsi verso il segno scritto. L'incertezza e il dubbio vengono resi espliciti dalle due domande con cui Dacia Maraini conclude il suo romanzo :

«Uscire dalla malia delle voci, come dice Adele Sòfia, ed entrare nella logica geometrica dei segni scritti ? Sarà un atto di saviezza o una scappatoia per eludere i corpi occhiuti e chiacchierini delle voci ? » (301).

Non riceviamo nessuna risposta a queste domande e, contrariamente al caso dell'omicidio di Angela Bari, la questione voci o scrittura rimane irrisolta, e lascia inquieto un lettore attento, rafforzando l'intuizione che il tema poliziesco non è l'unico argomento del libro. Nella lacerazione della protagonista la quale si dibatte tra il lasciare l'indeterminato e soggettivo universo della percezione per il rigoroso sistema della lingua scritta si nascondono interrogativi di lunghissima tradizione sui rapporti tra scrittura e percezione, ovvero tra la scrittura e il mondo al quale corporalmente apparteniamo.

La posizione della Maraini ci viene chiarita in un'altra opera, nel libro Amata scrittura dove la scrittrice cerca di conciliare l'inconciliabile cioè la scrittura e i sensi. Dice : «Scrivere vuol dire mettersi in rapporto con gli altri. Si scrive come si tende una mano [...] ma scrivere vuol dire prima di tutto dare un nome alle cose » (MARAINI, 2005 : 55). Con queste parole si riferisce all'atto di Adamo di cui parla Michel Foucault: "Sous sa forme première, quand il fut donné aux hommes par Dieu lui-même, le langage était un signe des choses absolument certain et transparent, parce qu'il leur ressemblait. [...] Et ces mots qu'Adam avait prononcés en les imposant aux animaux, ils sont demeurés, au moins en partie, emportant avec eux dans leur épaisseur, comme un fragment de savoir silencieux, les propriétés immobiles des êtres [...] » (FOUCAULT, 1966 : 51). Scrivere vuol dire riprodurre quell'antico atto di Adamo di dare un nome a una cosa, atto di padronanza e di conoscenza, vuol dire ricercare quella trasparenza perduta tra le parole e le cose. Sulla stessa linea concettuale si muove Italo Calvino il quale, in una delle sue lezioni americane, ribadisce il valore conoscitivo della sua scrittura e della lingua in generale. Secondo lui la parola unisce una traccia visibile con una cosa invisibile, con una cosa assente, che sia desiderata o temuta. Il giusto uso della lingua permette di avvicinarsi alle cose e a ciò che comunicano senza parole (CALVINO, 1996 : 69). D'altra parte, però, la Maraini ritorna sulla questione dei sensi : «Il lettore lo si raggiunge attraverso i sensi, non attraverso le enunciazioni » (MARAINI, 2005 : 61), perché l'uomo è un essere sensibile : comunica con gli altri e si collega con il mondo altrettanto sensibile attraverso i sensi, è un sensorium comune (cfr. MERLEAU-PONTY, 2003 : 283-322). Quindi l'udito, il senso protagonista del romanzo Voci come pure la vista, l'olfatto, il tatto, il gusto sono strumenti di conoscenza anche essi. Sembra che sia proprio il valore epistemologico a collegare la percezione uditiva e la parola scritta, la voce e la scrittura : entrambe hanno la stessa vocazione. 
La scrittura dunque, in quanto determinato sistema di segni intelligibili, a cui Dacia Maraini attribuisce un ruolo conoscitivo deve concordare con la natura sensuale e intelligibile della conoscenza percettiva dell'uomo, antecedente alla parola, la quale gli permette di intuire il senso delle cose al di fuori della lingua : scrivere e nello stresso tempo non « uscire dalla malia delle voci », usando le parole della Maraini. Tale scrittura avrebbe forse l'abilità di risalire agli strati archeologici della «chora semantica » (KRISTEVA, $1974: 22$ ) dell'essere, secondo quanto ci insegna Julia Kristeva (KRISTEVA, 1974 : 22-30).

\section{BIBLIOGRAFIA}

Per il testo di Dacia Maraini si fa riferimento all'edizione :

MARAINI, Dacia (2004), Voci, Milano, Biblioteca Superpocket.

La prima edizione dell'opera è :

MARAInI, Dacia (1994), Voci, Milano, Rizzoli.

\section{ALTRE OPERE}

ACKERMAN, Diane (1994), Historia naturalna zmystów, Warszawa, Książka i Wiedza (titolo originale : A Natural History of the Senses).

BARTHES, Roland, HAVAS Roland (1977), Ascolto, in : Enciclopedia Einaudi, Torino, Einaudi, vol. 1, s.v.

CACCIARI, Cristina (2002), Il rapporto fra percezione e linguaggio attraverso la metafora, consultabile sul sito web:

http://www.sssub.unibo.it/documenti/seminaria_metafora/cacciari.doc.

CALVINO, Italo (1996), Wykłady amerykańskie, Gdańsk-Warszawa-Marabut, Volumen (titolo originale : Lezioni americane).

ChangeuX, Jean-Pierre (1983), L'Homme neuronal, Paris, Fayard ; trad. ital. (1986) L'uomo neuronale, Milano, Feltrinelli.

CZAPLIŃSKI, Przemysław (1999), Rzecz w literaturze albo proza lat dziewięćdziesiatych wobec "mimesis", in : WYSŁOUCH, Seweryna e KAMIEŃSKA, Bogumiła (a cura di), Człowiek i rzecz. O problemach reifikacji w literaturze, filozofii i sztuce, Poznań, Poznańskie Studia Polonistyczne, Seria Literacka.

DERRIDA, Jacques (1997), Gtos i fenomen. Wprowadzenie do problematyki znaku $w$ fenomenologii Husserla (titolo originale : La voix et la fénomène), Warszawa, Wydawnictwo KR.

Di PAOLO, Paolo (2003), Le stagioni di Dacia Maraini. Una lettura critica dell'opera della scrittrice, Milano, italialibri.net, http://www.italialibri.net/dossier/maraini/stagioni.html.

DUFRENNE, Mikel (1987), L'oeil et l'oreille, Montréal, L'Hexagone.

FLIEGER, Hanna (1994), Il rapporto varianti/costanti nella poetica di Italo Calvino. Modalità attenzionali e implicazioni culturali, Poznań-Toruń, Wydawnictwo Edytor.

FOUCAULT, Michel (1966), Les mot et les choses, Paris, Editions Gallimard. KRISTEVA, Julia (1974), La révolution du language poétique, Paris, Editions du Seuil. 
MARAINI, Dacia (2005), Amata scrittura, Milano, Bur.

MARAINI, Dacia (2004), Bagheria, Milano, Bur.

MARAINI, Dacia (1998), Il viaggio per scoprire se stessi, Il grillo, [10/3/1998] http://www.emsf.rai.it/grillo/trasmissioni.asp?d=198

MARAINI, Dacia (1997), Da un'intervista inedita,

http://rizzoli.rcslibri.corriere.it/rizzoli/_minisiti/maraini_/ita/inter/intro.htm.

MERLEAU-PONTY, Maurice (2003), Fenomenologia della percezione (titolo originale : Phénoménologie de la perception), Milano, Bompiani.

MiLler, Glenn, Johnson-LAIRD Phlip (1976), Language and Perception, Cambridge, Cambridge University Press.

ONG, Walter J. (1986), Oralità e scrittura (titolo originale: Orality and Literacy. The Technologizing of The Word), Bologna, Il Mulino.

ROSI, Viviana (2005), Introduzione a : MARAINI Dacia, Amata scrittura, Milano, Bur.

SCHOLEM, Gershom (1970), Il nome di Dio e la teoria cabalistica del linguaggio, Milano, Adelphi.

SERRES, Michel (1985), Les cinq sens. Philosophie des corps mêlé, Paris, Bernard Grasset.

TABUCCHI, Antonio (2003), Autobiografie altrui. Poetiche a posteriori, Milano, Feltrinelli.

WILKOSZEWSKA, Krystyna (1998), W krainie czucia i bezczucia, czyli o zmysłach głównych, zapomnianych i sztucznych, in : Wymiary piękna. Z badań estetyki sensu largo, a cura di M. GoŁASZEWSKA, Kraków, Wydawnictwo UJ.

\begin{abstract}
This article, which is devoted to Dacia Maraini's novel Voci, analyses the sound and auditory aspect of a literary text. A special advantage and meaning of the experiences of the sense of hearing manifests itself in the main character's unique experience of reality by means of the audible : voices, noises and sounds. Presence or absence of the voice and of the body which produces them in time and space, build a metaphorical structure that stretches between life and death in the novel. The sense of hearing is also a cognitive instrument of the surrounding reality which emerges through the voices. Getting to know the truth becomes possible only via the voice. Acoustics of the novel make readers reflect upon the unsolvable friction between orality and the written word.
\end{abstract}

